\title{
A fenestrated stent graft for surgical resection of lung cancer invading the aortic arch
}

\author{
Toshiyuki Nagata, MD, ${ }^{\text {a }}$ Yoshihiro Nakamura, MD, $\mathrm{PhD},{ }^{\mathrm{a}}$ Hiroyuki Yamamoto, $\mathrm{MD}, \mathrm{PhD},{ }^{\mathrm{b}}$ and \\ Masami Sato, MD, PhD, ${ }^{\text {a }}$ Kagoshima, Japan
}

A fenestrated stent graft that included the orifice of the left subclavian artery was used to resect a left upper lobe cancer invading the distal aortic arch without the use of cardiopulmonary bypass. No complications were observed. We found no previous reports in our literature search of fenestrated stent-graft placement for distal aortic arch tumor invasion. This method could reduce the risks of combined resection of the aortic arch during lung cancer surgery.

\section{CLINICAL SUMMARY}

A 67-year-old man had an abnormal computed tomographic shadow detected during follow-up after subtotal esophageal resection through a right thoracotomy 9 years before. The pathologic diagnosis was p-stage I squamous cell carcinoma. Primary lung cancer with aortic invasion was suspected on computed tomography, which showed a 23-mm diameter nodule in the left lung segmentum apicodorsale $15 \mathrm{~mm}$ distal to the orifice of the left subclavian artery. The nodule's border with the aorta was unclear across a length of $23 \mathrm{~mm}$, corresponding to a third of the aortic circumference (Figure 1, $A$ ). Bronchoscopic biopsy failed to provide a definitive diagnosis. There were no radiologic signs of other malignancies on whole-body computed tomography and positron emission tomography. Transesophageal ultrasonography was not conducted to clarify aortic wall invasion because of the patient's postoperative state. The patient was considered to have cT4NOM0 cancer and was deemed a candidate for complete resection. After consideration of surgical morbidity and mortality, we chose the fenestrated stent method rather than replacement of the aortic arch under cardiopulmonary bypass.

First, the patient underwent video-assisted thoracoscopic surgery, which led to the diagnosis of adenocarcinoma with

\footnotetext{
From the Department of General Thoracic Surgery, ${ }^{\mathrm{a}}$ Graduate School of Medical and Dental Sciences Kagoshima University, Kagoshima, Japan; and the Department of Cardiovascular and Gastroenterological Surgery, ${ }^{\text {b }}$ Advanced Therapeutics, Cardiovascular and Respiratory Disorders, Graduate School of Medical and Dental Sciences, Kagoshima University, Kagoshima, Japan.

Disclosures: Authors have nothing to disclose with regard to commercial support.

Received for publication Nov 29, 2012; revisions received Feb 14, 2013; accepted for publication Feb 28, 2013; available ahead of print March 27, 2013.

Address for reprints: Masami Sato, MD, PhD, Department of General Thoracic Surgery, Graduate School of Medical and Dental Sciences Kagoshima University, 8-35-1 Sakuragaoka, Kagoshima, 890-8520, Japan (E-mail: m-sato@m2.kufm. kagoshima-u.ac.jp).

J Thorac Cardiovasc Surg 2013;146:238-9

0022-5223/ $\$ 36.00$

Copyright (C) 2013 by The American Association for Thoracic Surgery http://dx.doi.org/10.1016/j.jtcvs.2013.02.070
}
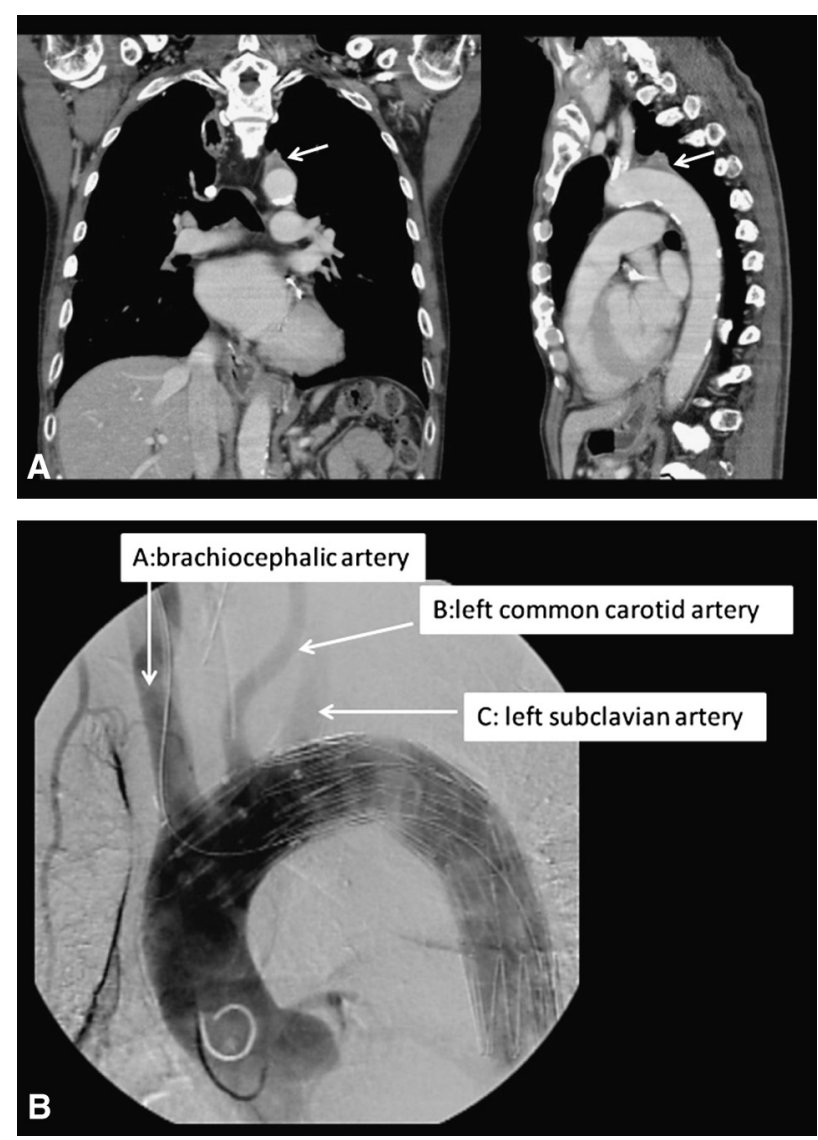

FIGURE 1. A, Computed tomographic scan coronal (left) and sagittal (right) views of tumor (arrows) attached to the distal portion of the aortic arch, $23 \mathrm{~mm}$ in length and a third of the circumference. Note the unclear tumor margin. B, Fluorography during stent operation. A customized fenestrated stent was inserted into the target zone of the aorta. Angiograms revealed patency of both the brachiocephalic artery $(A)$ and left common carotid artery $(B)$ without endoleak. Delayed pooling of the left subclavian artery $(C)$ was seen as a result of backflow from the cerebral circulation.

direct tumor invasion to the mediastinum. To gain a sufficient margin from the tumor and landing zone, we ordered a customized fenestrated stent graft that would open only to the right brachiocephalic artery and left common carotid artery and would cover the left subclavian artery. We started preoperative radiotherapy. After informed consent was obtained, a thoracic endovascular fenestrated stent graft (Najuta $\Phi 30 \times 200 \mathrm{~mm}$; Kawasumi Laboratories Inc, Tokyo, Japan) was placed 25 days after the initial video-assisted thoracoscopic surgery (Figure 1, B). The 


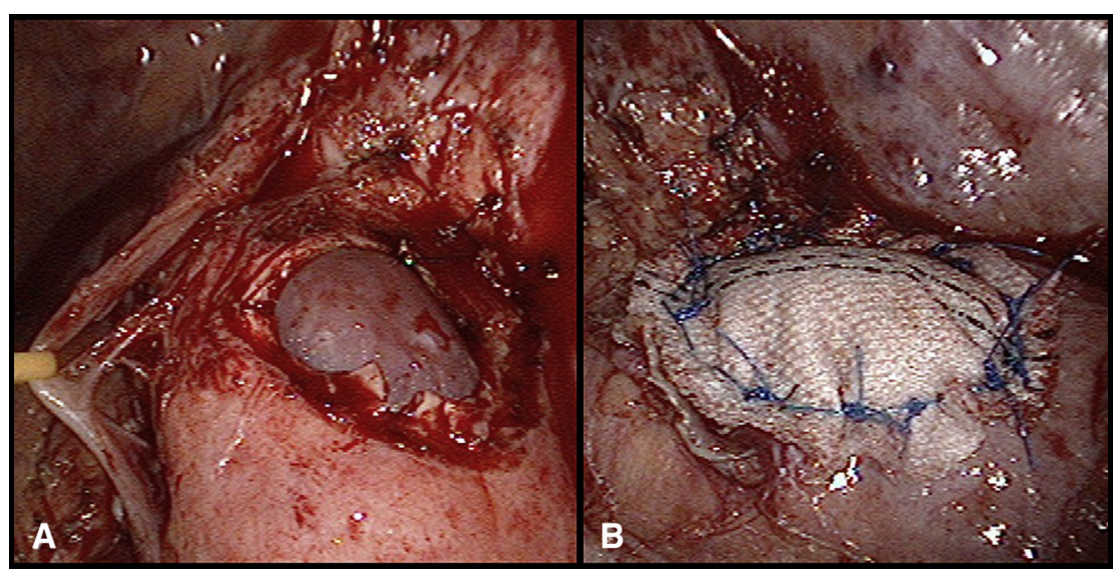

FIGURE 2. Intraoperative views. A, The stent can be seen through the defect of the aortic wall. No bleeding was observed during the procedure. B, A Hemashield fabric patch was used to close the defect.

procedure was performed under general anesthesia through a retrograde brachial and femoral approach.

Two weeks after radiotherapy, pulmonary resection with combined resection of the aortic wall was carried out. The left upper lobe was divided in two because of immobility of the invaded site, and left upper lobectomy was performed. The left upper lobe bronchial stump, covered by intercostal muscle and lymph nodes, was systematically dissected. These procedures opened the operating field to allow aortic clamping if necessary. The aortic wall was dissected in full thickness at least $5 \mathrm{~mm}$ beyond the tumor invasion margin without cardiopulmonary bypass. During this procedure, the inserted stent was seen but bleeding was not observed (Figure 2, A). The defect of the aortic wall $(30 \times 30 \mathrm{~mm})$ was closed with a $37.5 \times 37.5-\mathrm{mm}$ Hemashield fabric patch (Maquet $\mathrm{GmbH}$ \& Co KG, Rastatt, Germany) with 12 Prolene 4-0 (Ethicon, Inc, Somerville, NJ) mattress sutures (Figure 2, $B$ ).

The postoperative course was uneventful. Histologic examination revealed a T4NOM0 adenocarcinoma invading the aortic adventitia; however, the aortic margin was cancer free. The patient was discharged on postoperative day 15 . He received adjuvant chemotherapy. Local recurrence and distant metastasis have not been observed as of 16-month follow-up. Complications such as migration and aneurysm have also not been observed.

\section{DISCUSSION}

Surgical resection of T4 non-small cell lung cancer invading the large vessels remains controversial. A lack of randomized studies combined with only a small number of case series means that there is a low evidence level for this surgical resection type. Some reports, however, do suggest that complete resection for T4N0-1M0 disease has a favorable prognosis with long-term survival. ${ }^{1}$

With combined aortic resection, a side clamp or crossclamp is usually used. Furthermore, to avoid organ ischemia, permanent or temporary graft bypass or extracorporeal circulation with cardiopulmonary bypass is necessary. Because of potential risks, indications for these procedures should be firmly restricted. ${ }^{2}$

In this case, we focused on a procedure to remove the aortic wall with an aortic endograft. Replacement with artificial vessels has been the main method to date; however, the use of endovascular surgery, which has low invasiveness, is increasing. In cases involving aortic arch aneurysm or distal arch aneurysm, the stent-graft method is difficult because preservation of the arch branch bloodstream is necessary. Branched or fenestrated stent grafting is therefore an expected future development. ${ }^{3}$

\section{CONCLUSIONS}

Treatment of lung cancer cases with descending aortic involvement by combined resection of the aorta with stent grafting have been reported previously $y^{4,5}$; however, this is the first report describing the use of a fenestrated stentgraft. Fenestrated or branched stent grafts could reduce perioperative risk and allow complete resection in patients with invasion of the aorta, especially the aortic or distal arch.

\section{References}

1. Ohta M, Hirabayasi H, Shiono H, Minami M, Maeda H, Takano H, et al. Surgical resection for lung cancer with infiltration of the thoracic aorta. $J$ Thorac Cardiovasc Surg. 2005;129:804-8.

2. de Perrot M, Fadel E, Mussot S, de Palma A, Chapelier A, Dartevelle P. Resection of locally advanced (T4) non-small cell lung cancer with cardiopulmonary bypass. Ann Thorac Surg. 2005;79:1691-7; discussion 1697.

3. Kawaguchi S, Yokoi Y, Shimazaki T, Koide K, Matsumoto M, Shigematsu H Thoracic endovascular aneurysm repair in Japan: experience with fenestrated stent grafts in the treatment of distal arch aneurysms. J Vasc Surg. 2008;48(6 Suppl): 24S-9S; discussion 29S.

4. Berna P, Bagan P, De Dominicis F, Dayen C, Douadi Y, Riquet M. Aortic endosten followed by extended pneumonectomy for T4 lung cancer. Ann Thorac Surg. 2011;91:591-3.

5. Marulli G, Lepidi S, Frigatti P, Antonello M, Grego F, Rea F. Thoracic aorta endograft as an adjunct to resection of a locally invasive tumor: a new indication to endograft. J Vasc Surg. 2008;47:868-70. 